\title{
Beobachtungen des Cometen 1893 II
}

am Refractor (244 $\mathrm{mm}$ Oeffnung) der Königl. Sternwarte in Berlin.

\begin{tabular}{|c|c|c|c|c|c|c|c|c|c|c|}
\hline 1893 & M. Z. Berlin & $\Delta \alpha$ & $\Delta d$ & Vgl. & $\alpha$ app. & $\log p \cdot \Delta$ & $\delta$ app. & $\log p \cdot \Delta$ & Red.ad l.app. & * \\
\hline Juli $\quad$ I 8 & $10^{\mathrm{h}} \quad 6^{\mathrm{m}} \mathrm{I1}^{\mathrm{s}}$ & $+1^{\mathrm{m}} \mathrm{I} 0^{\mathrm{s}} \cdot 78$ & $\cdots 5^{\prime} 16 . " 7$ & 0 & II ${ }^{\mathrm{h}} \quad 4^{\mathrm{m}} 59^{\mathrm{s}} \cdot 5^{8}$ & 12 & $+30^{\circ} 13^{\prime} 22^{\prime \prime} 4$ & 0.823 & $+0^{5} \cdot 74+4.7$ & 6 \\
\hline 20 & $\begin{array}{lll}9 & 42 & 44\end{array}$ & -033.76 & -250.6 & 18.6 & II $20 \quad 29.58$ & 9.608 & +265612.7 & 0.810 & $+0.78+3.5$ & 8 \\
\hline
\end{tabular}
verschwand.

Juli I8. Beobachtung sehr mangelhaft wegen Störungen durch Wolken, in welchen der Comet sehr bald

Bestimmungen von Vergleichsternen für Comet 1893 II am grossen Berliner Meridiankreise.

(Mittleres Aequinoctium 1893.0, Epoche 1894.2.)

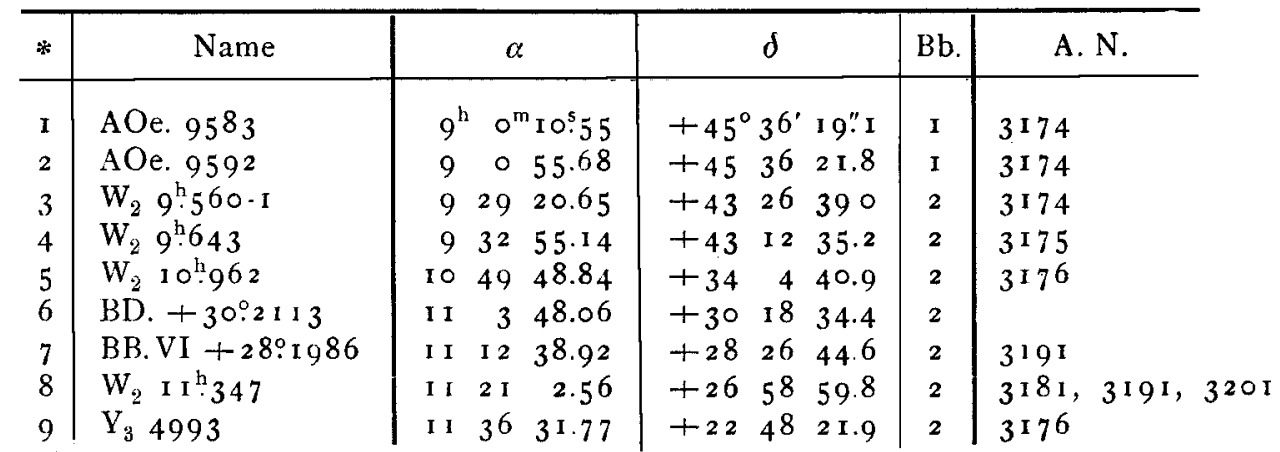

Unter letzter Columne ist der Ort angegeben, an welchem der Stern unter der Bezeichnung der Col. 2 benutzt wurde. Der mittlere zufällige Fehler einer Beobachtung beträgt \pm 0.028 (in Bogen grössten Kreises \pm 0.36 ), resp. $\pm 0 . " 27$.

Berlin 1894 Juli 15.

H. Battermann.

Beobachtungen des Cometen 1894 I (Denning) und der Nova (T) Aurigae am ro $\frac{1}{2} z$ ölligen Refractor der k. Sternwarte zu München von K. Oertel.

Comet 1894 I (Denning).

\begin{tabular}{|c|c|c|c|c|c|c|c|c|c|c|c|}
\hline 1894 & M.Z.Münch. & $\Delta \alpha$ & $\Delta \delta$ & Vgl. & & $\alpha$ app. & $\log p .4$ & $\delta$ app. & $\log p .4$ & Red. ad 1. app. & $*$ \\
\hline ärz 28 & $8^{\mathrm{h}} 18^{\mathrm{m}} 37^{\mathrm{s}}$ & $+\mathrm{I}^{\mathrm{m}} 35^{\mathrm{s}} \cdot 04$ & $-4^{\prime} 43^{\prime \prime} 8$ & I 8.6 & $10^{b}$ & $\mathrm{I}^{\mathrm{m}} 47^{\mathrm{s}} \cdot 12$ & $9 \cdot 196 n$ & $+31^{\circ} 10^{\prime} 37^{\prime \prime} 5$ & $0.43^{6}$ & $+2.08+1.0$ & I \\
\hline 29 & $\begin{array}{lll}8 & \text { I } 4 & 43\end{array}$ & +021.32 & $-2 \quad 32.8$ & 30.10 & IO & 522.55 & $9.207 \mathrm{n}$ & +303741.1 & $0.45^{\circ}$ & $+2.07+0.6$ & 3 \\
\hline 30 & 81010 & to $35.3 \mathrm{I}$ & +147.3 & $\mathrm{I} 8.6$ & Io & $8 \quad 52.68$ & $9 \cdot 354 \mathrm{n}$ & $+30 \quad 5 \quad 0.7$ & 0.464 & $+2.06+0.3$ & 5 \\
\hline $3^{\mathrm{I}}$ & $\begin{array}{lll}8 & 33 & 21\end{array}$ & $-220.8 \mathrm{r}$ & +3 I 4.4 & 18.6 & 10 & $12 \quad 20.33$ & $9.243 n$ & +293145.7 & $0.4^{6} 5$ & $+2.06-0.2$ & 6 \\
\hline April I & $\begin{array}{lll}8 & 20 & 38\end{array}$ & +o 44.54 & -329.2 & I 8.6 & 10 & $15 \quad 37.49$ & $9.16 I_{n}$ & $\begin{array}{r}+285935.4 \\
\end{array}$ & 0.480 & $+2.05-0.3$ & 8 \\
\hline 2 & 83319 & to 34.13 & -546.4 & 24,8 & 10 & $18 \quad 52.74$ & $9.072 \mathrm{n}$ & +282659.1 & 0.486 & $+2.04-0.5$ & IO \\
\hline 3 & $\begin{array}{lll}8 & 24 & 37\end{array}$ & to 29.80 & $\ldots 042.5$ & 24.8 & ro & $22 \quad 1.27$ & $9.137 \mathrm{n}$ & +2755 10.1 & 0.500 & $+2.01-0.5$ & I 2 \\
\hline 7 & $\begin{array}{lll}9 & 47 & 6\end{array}$ & to 27.99 & - & 31.0 & 10 & 3355.66 & $8.908 \mathrm{n}$ & - & - & +1.99 & $14^{*}$ \\
\hline 7 & $\begin{array}{lll}9 & 53 & 3\end{array}$ & - & +3369 & 0.7 & & - & - & +2548526 & 0.526 & $-\mathrm{I} .7$ & I 4 \\
\hline
\end{tabular}

Mittlere Oerter der Vergleichsterne für 1894.0.

\begin{tabular}{|c|c|c|c|c|c|c|c|c|c|}
\hline$*$ & & $\alpha$ & & $\delta$ & Autorität & $*$ & $\alpha$ & $\delta$ & Autorität \\
\hline I & $10^{\mathrm{h}} \circ$ & $0^{\mathrm{m}} 10.00$ & $+31^{\circ} 1$ & $5^{\prime} 20^{\prime \prime} 3$ & AG. Leid. Z. 163,286 & 8 & $10^{h} 14^{m} 50.90$ & $+29^{\circ} 3^{\prime} 4^{\prime \prime} 8$ & Anschl. \\
\hline 2 & Io 5 & $5 \quad 4094$ & +304 & $40 \quad 15.9$ & AG. Leid. Z. 37,39 & 9 & $\begin{array}{lll}\text { I0 } & \text { I } 5 & 27.97\end{array}$ & +283213.9 & BB. VI $+280^{\circ} 872$ \\
\hline 3 & 104 & $459 \cdot 16$ & +304 & $40 \quad 13.3$ & Anschl. an $* 2(18.6 \mathrm{Bb}$.) & 10 & I0 $18 \quad 16.57$ & $+283^{2} 46.0$ & Anschl. an $* 9$ ( $12.4 \mathrm{Bb}$.) \\
\hline 4 & 105 & $5 \quad 47.74$ & +295 & 5948.3 & AG. Leid. Z. 37,39 & I I & $10 \quad 20 \quad 14.28$ & +275037.1 & Bess. Z. 526 \\
\hline 5 & 108 & $8 \times 5.31$ & +30 & $\begin{array}{lll}3 & 13.1\end{array}$ & Anschl an $* 4(12.4 \mathrm{Bb})$. & I 2 & $10 \quad 2129.46$ & +275553.1 & Anschl. an $*$ I I (12.4 Bb. $)$ \\
\hline 6 & 1014 & 439.08 & $+292 \xi-1$ & 831.5 & Paris 12654 & I 3 & 103131.86 & +253754.7 & Paris 13002 \\
\hline 7 & $10 \quad 15$ & $15 \quad 29.88$ & +29 & $4 \quad 13.3$ & Paris 12665 & 14 & I0 $33 \quad 25.68$ & +2545 1 7.4 & Anschl. an $* 13(\mathrm{I} 2.4 \mathrm{Bb})$ \\
\hline
\end{tabular}

